\title{
Neoplasia Quística Mucinosa Gigante del Páncreas Tratada con Pancreatectomía Corporocaudal y Preservación Esplénica
}

\author{
Giant Mucinous Cystic Neoplasm of the Pancreas \\ Undergoing Pancreatectomy with Splenic Preservation
}

Carlos Manterola ${ }^{1,2}$; Andrés Navarrete ${ }^{2}$ \& Renato Becker ${ }^{3}$

\begin{abstract}
MANTEROLA, C.; NAVARRETE,A. \& BECKER, R. Neoplasia quística mucinosa gigante del páncreas tratada con pancreatectomía corporocaudal y preservación esplénica. Int. J. Morphol., 38(6):1722-1728, 2020.

RESUMEN: La neoplasia quística mucinosa del páncreas (NQMP) es un tumor infrecuente, que afecta predominantemente a mujeres (90-95\%), afectando especialmente entre la quinta y séptima década de la vida; cuyo descubrimiento suele ser incidental. Por lo general, son lesiones solitarias, sin compromiso del conducto pancreático principal; poco sintomáticas y asociadas a malignidad (10 \% a $40 \%$ ). El objetivo de este manuscrito fue reportar un caso de neoplasia quística mucinosa gigante del páncreas intervenida quirúrgicamente y revisar la evidencia existente respecto de sus características morfológicas, terapéuticas y pronósticas. Mujer de 29 años, con masa abdominal poco sintomática. El diagnóstico se verificó por medio de ultrasonografía, tomografía axial computarizada y resonancia nuclear magnética. Se intervino quirúrgicamente, realizándose pancreatectomía corporocaudal con preservación esplénica, sin incidentes. La paciente fue dada de alta al quinto día, sin complicaciones, y evolucionó de forma adecuada, sin complicaciones postoperatorias. La NMQP es una lesión compleja, que puede asociarse a malignidad, pero el diagnóstico preoperatorio de malignidad no puede establecerse con seguridad. El pronóstico depende de un diagnóstico precoz y un tratamiento oportuno.
\end{abstract}

\section{PALABRAS CLAVE: Quiste pancreático; Neoplasia pancreática; Pancreatectomía.}

\section{INTRODUCCIÓN}

La neoplasia quística mucinosa del páncreas (NQMP) es un tumor infrecuente, que afecta predominantemente a mujeres (90-95\%), afectando especialmente entre la quinta y séptima década de la vida; cuyo descubrimiento suele ser incidental por cortes imagenológicos del abdomen. Por lo general, son lesiones solitarias, sin compromiso del conducto pancreático principal; poco sintomáticas y asociadas a malignidad en un 10 a $40 \%$ de los casos, dependiendo en especial de su tamaño (Jorba et al., 2008; Troncoso et al., 2015; Burk et al., 2018; European Study Group on Cystic Tumours of the Pancreas, 2018; van Huijgevoort et al., 2019; Hurtado-Pardo et al., 2019; Hasan et al., 2019).

Desde el punto de vista patológico, la NQMP suele estar formada por quistes de más de $2 \mathrm{~cm}$, en cuyo interior existen septos y mucina. En ocasiones son multiloculares, con hasta 6 quistes, pero sin comunicación con sistema ductal pancreático. Puede presentarse como tumor único macroquiste con proyecciones papilares y calcificaciones periféricas en "cáscara de huevo", que son sugerentes de malignidad. Son de tipo epitelial y suelen estar compuesta por epitelio columnar, productor de mucina con diversos grados de displasia (Jorba et al.; Terris, 2011).

Existen algunas guías clínicas y artículos de recomendación de expertos, que han propuesto algoritmos para la toma de decisiones terapéuticas en pacientes con lesiones quísticas mucinosas del páncreas en general, que incluyen algunas situaciones de las NQMP (Tanaka et al., 2012; Vege et al., 2015; Basturk et al., 2015; European Study Group on Cystic Tumours of the Pancreas; van Huijgevoort et al.).

El tratamiento de la NQMP, según su tamaño, suele ser quirúrgico. Sin embargo, la morbilidad postoperatoria menor incluso en centros de referencias se ha reportado hasta en el 23,1\% de los casos. Además, existe evidencia de morbilidad postoperatoria mayor como peritonitis y sepsis de $13,3 \%$ y $12,2 \%$ respectivamente; un índice de reintervenciones de hasta $15,8 \%$; y de mortalidad asociada a resección pancreática distal con y sin preservación

\footnotetext{
${ }^{1}$ Centro de Excelencia en estudios Morfológicos y Quirúrgicos (CEMyQ), Universidad de La Frontera, Temuco, Chile.

${ }^{2}$ Clínica RedSalud Mayor, Temuco, Chile.

${ }^{3}$ Instituto de Patología Celular y Molecular (iPat), Temuco, Chile.
} 
esplénica de 7,3\% y 7,7 \% respectivamente (Nimptsch et al., 2016).

El objetivo de este manuscrito fue reportar un caso de neoplasia quística mucinosa gigante del páncreas intervenida quirúrgicamente y revisar la evidencia existente respecto de sus características morfológicas, terapéuticas y pronósticas.

\section{PRESENTACIÓN DEL CASO}

Este reporte, fue escrito siguiendo la guía Case Report Guidelines (CARE) (Gagnier et al., 2014).

Se trata de una paciente de 29 años de edad, de sexo femenino, derivada por su médico tratante por hallazgo de masa abdominal poco sintomática en exploración abdominal en un examen de rutina. Se solicitó tomografía axial computarizada (TAC) y resonancia nuclear magnética (RNM), las que permitieron evidenciar la presencia de una gran lesión quística de la cola del páncreas, de aproximadamente 8,4 x 8,8 x $11 \mathrm{~cm}$ de diámetro. Presentaba una pared bien definida, con un espesor de hasta $4 \mathrm{~mm}$ y discreto realce con el contraste. Se identificaron múltiples septos finos, algo irregulares, principalmete en su aspecto superior y medial; con un realce apenas perceptible con el contraste, pero sin elementos nodulares sólidos. Presentaba además, una lobulación de sus contornos en su aspecto inferior, con zonas de proyección exofítica de áreas quísticas respecto a la lesión principal. El resto de los segmentos del páncreas se encontraban de forma y señal normales. El conducto pancreático principal era fino. El estómago se encontraba deplazado hacia anterior y superior, en contacto con la lesión (Figs. 1 y 2). De
Tabla I. Pruebas de laboratorio preoperatorias.

\begin{tabular}{|c|c|c|}
\hline Variables & Caso clínico & Valores normales \\
\hline Hemoglobina $(\mathrm{g} / \mathrm{dL})$ & 13,4 & $13,5-17,0$ \\
\hline Hematocrito ( \%) & 37,0 & $40-54$ \\
\hline Leucocitos $\left(10^{\wedge} 3 / \mathrm{ul}\right)$ & 8,20 & $4000-10000$ \\
\hline Plaquetas $\left(10^{\wedge} 3 / \mathrm{ul}\right)$ & 240 & 1 \\
\hline VHS (mm/hr) & 33,9 & $0-13$ \\
\hline Glicemia (mg/dL) & 89,0 & $70-100$ \\
\hline Creatinina $(\mathrm{mg} / \mathrm{dL})$ & 1,0 & $0,6-1,2$ \\
\hline Amilasa (U/L) & 34,0 & $12-70$ \\
\hline Lipasa (U/L) & 56,0 & $0-137$ \\
\hline Proteinas totales $(\mathrm{g} / \mathrm{dL})$ & 7,6 & $6,4-8,3$ \\
\hline Albúmina (g/dL) & 4,1 & $3,4-4,8$ \\
\hline Bilirubina total $(\mathrm{mg} / \mathrm{dL})$ & 1,0 & $0,2-1,3$ \\
\hline Fosfatasas alcalinas & 76,0 & $38-126$ \\
\hline ASAT (U/L) & 31,0 & $17-59$ \\
\hline ALAT (U/L) & 29,0 & $21-72$ \\
\hline GGTP (U/L) & 66,0 & $15-73$ \\
\hline Protrombina ( \%) & 81,3 & $70-100$ \\
\hline TTPK (seg) & 23,2 & $21-32$ \\
\hline CEA $(\mathrm{ng} / \mathrm{mL})$ & 1,2 & $0-3$ \\
\hline $\mathrm{Ca} 125(\mathrm{U} / \mathrm{mL})$ & 4,3 & $<35$ \\
\hline Ca $19-9(\mathrm{U} / \mathrm{mL})$ & 2,6 & $<37$ \\
\hline
\end{tabular}

CEA: Antígeno carcinoembrionario.

las pruebas de laboratorio, sólo llamaba la atención un incremento en la velocidad de eritrosedimentación (Tabla I). Con diagnóstico de tumor quístico-mucinoso gigante del páncreas, se indicó cirugía electiva.

Con profilaxis antibiótica, y bajo anestesia epidural y general con gases; la paciente fue intervenida quirúrgicamente. Una vez realizada la laparotomía, se evidenció una gran masa irregular, heterogénea, que protruía desde el páncreas, desplazando el estómago hacia adelante.
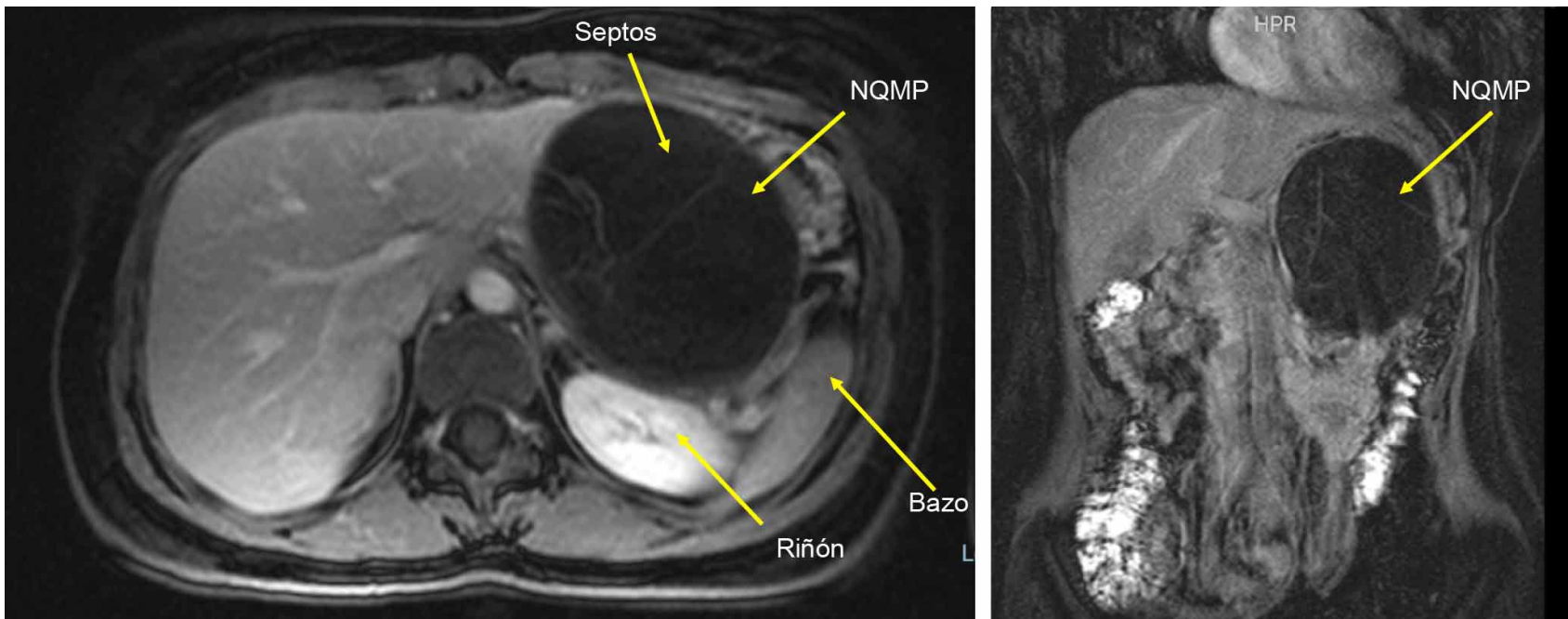

Fig. 1. TAC de abdomen: Reconstrucción axial y coronal, en la que se aprecia a nivel del cuerpo y cola del páncreas una gran lesión de hipodensa, redondeada, de contorno lisos, pared bien definida, con tabiques interiores que realzan levemente con uso de contraste intravenoso; y que mide 8,4 x 8,8 x $11 \mathrm{~cm}$ de diámetro. 

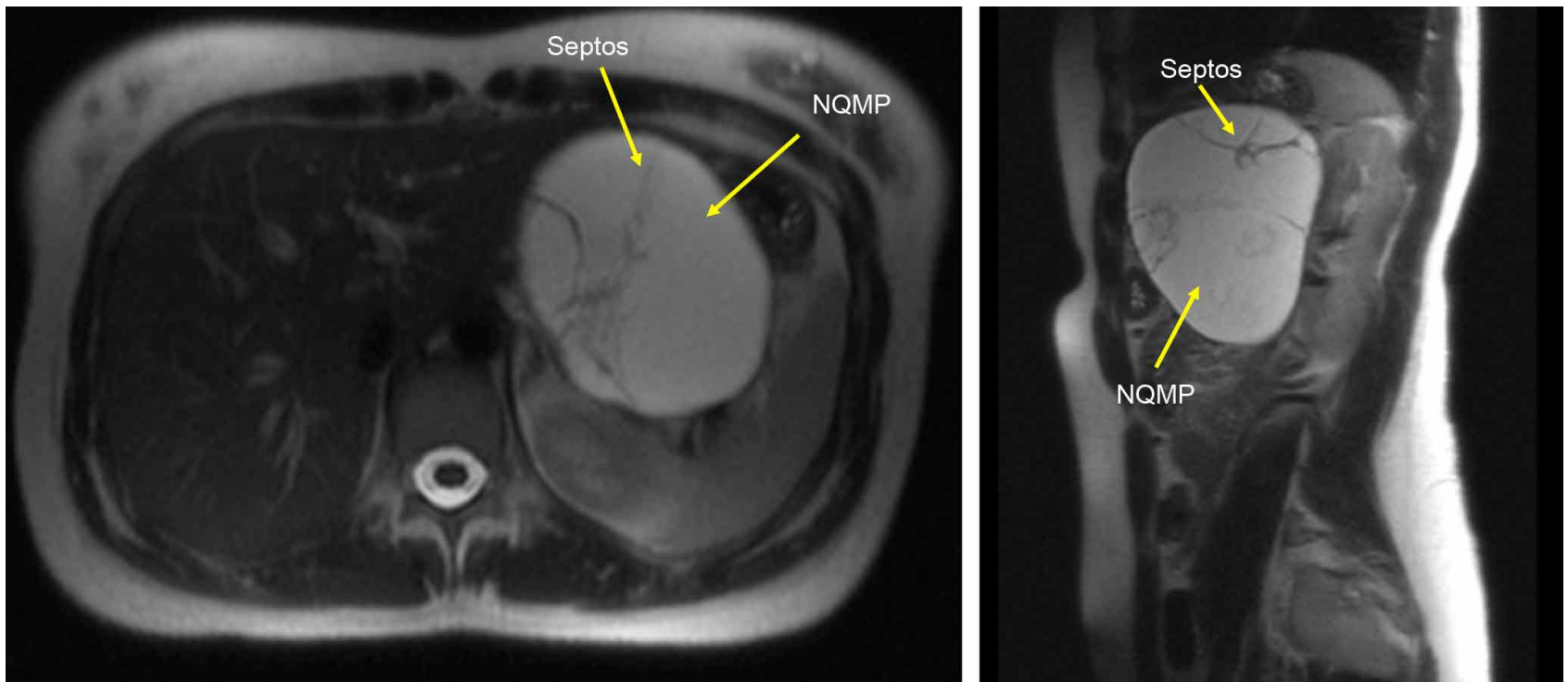

Fig. 2. RNM de abdomen: Reconstrucción axial y sagital, en la que se verifica en la unión de cuerpo y cola del páncreas, una lesión quística ovoidea, ligeramente lobulada, de paredes bien definidas y con tabiques internos algo irregulares, principalmete en su aspecto superior y medial.

Tras la apertura de la bolsa omental, se realizó preservación de la arcada arterial gastroomental y de los vasos cortos. Se seccionó el ligamento espleno-cólico y se realizó una suave disección de los márgenes inferior y superior del páncreas, disecando el plano retropancreático y la confluencia del eje venoso espleno-portal. Posteriormente, se seccionó el cuerpo pancreático con dispositivo Endo GIATM y dos cargas blancas de $40 \mathrm{~mm}$. Se disecaron las venas porta, mesentérica y esplénica (esta última comprimida por la lesión); y se procedió a la sección y ligadura de la vena esplénica. Se completó la pancreatectomía corporocaudal con preservación esplénica con técnica de Warshaw (Warshaw, 1988), sin incidentes (Fig. 3). El tiempo operatorio fue de 110 minutos, con una pérdida hemática total de $200 \mathrm{cc}$.
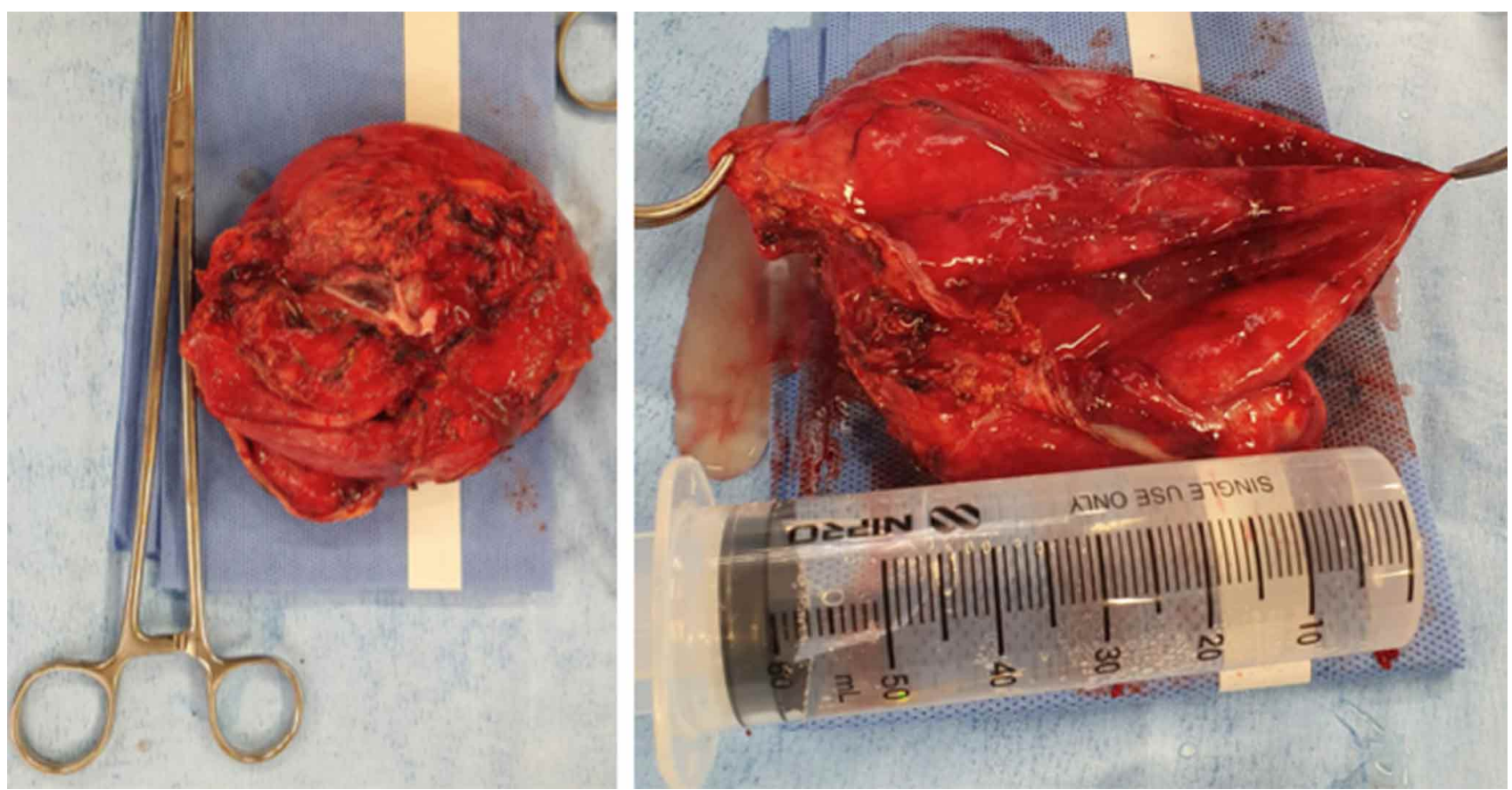

Fig. 3. Pieza quirúrgica en fresco. Se puede observar la lesión quística vacía, que alcanza $11 \mathrm{~cm}$ de diámetro mayor. 
Se dejó dren siliconado de 18 Fr en lecho pancreático, que tuvo un débito promedio de $55 \mathrm{cc} / 24$ horas, de aspecto serohemático oscuro (la medición de amilasas en este fluido fue de 7531 el primer día; para normalizarse al tercer día). La paciente evolucionó de forma satisfactoria, sin evidencias de fístula pancreática; por lo que fue dada de alta al $5^{\circ}$ día post operatorio.

El estudio histopatológico de la pieza quirúrgica fue concluyente de NQMP pancreática con displasia de alto grado, con carcinoma in situ y microfocos de invasión convencional (tamaño de los microfocos de invasión: menores a $5 \mathrm{~mm}$ ), sin compromiso extracapsular, con márgenes quirúrgicos negativos para neoplasia, sin invasión linfovascular ni perineural; por lo que quedó catalogado como pT1a Nx Mx (Figs. 4 y 5).

En reunión de Comité Oncológico, se decidió adyuvancia con quimioterapia dado el componente microinvasor, instaurándose tratamiento con FOLFIRINOX (FOL - Ácido folínico (leucovorina); F 5-fluorouracilo (5-FU); IRIN - Irinotecán; OX Oxaliplatino), un ciclo cada 15 días. En sus controles posteriores, la paciente ha estado asintomática (después de cuatro meses de seguimiento se encontraba realizando su adyuvancia sin incidentes).
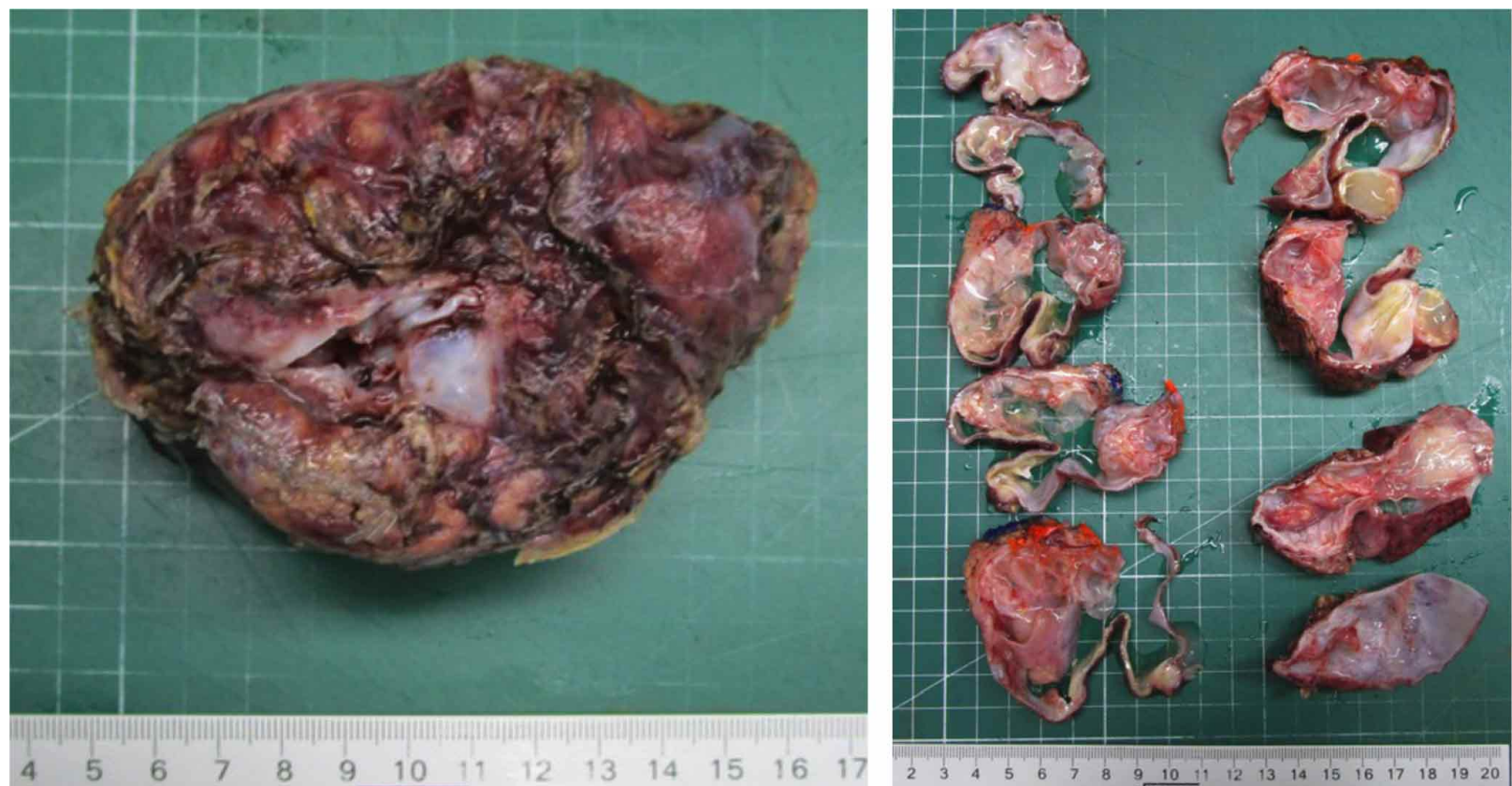

Fig. 4. Pieza quirúrgica fijada y múltiples cortes que permiten ver áreas quísticas y depósitos de mucina. La lesión es de 9,7 x 5,5 x 5,8 $\mathrm{cm}$ de ejes mayores de superficie pardo-rosada con petequias, de márgenes bien delimitados, quístico multilocular con abundante material mucinoso. En una de sus caras, se reconoce fragmento compatible con tejido pancreático en un área de $3 \times 2 \mathrm{~cm}$.
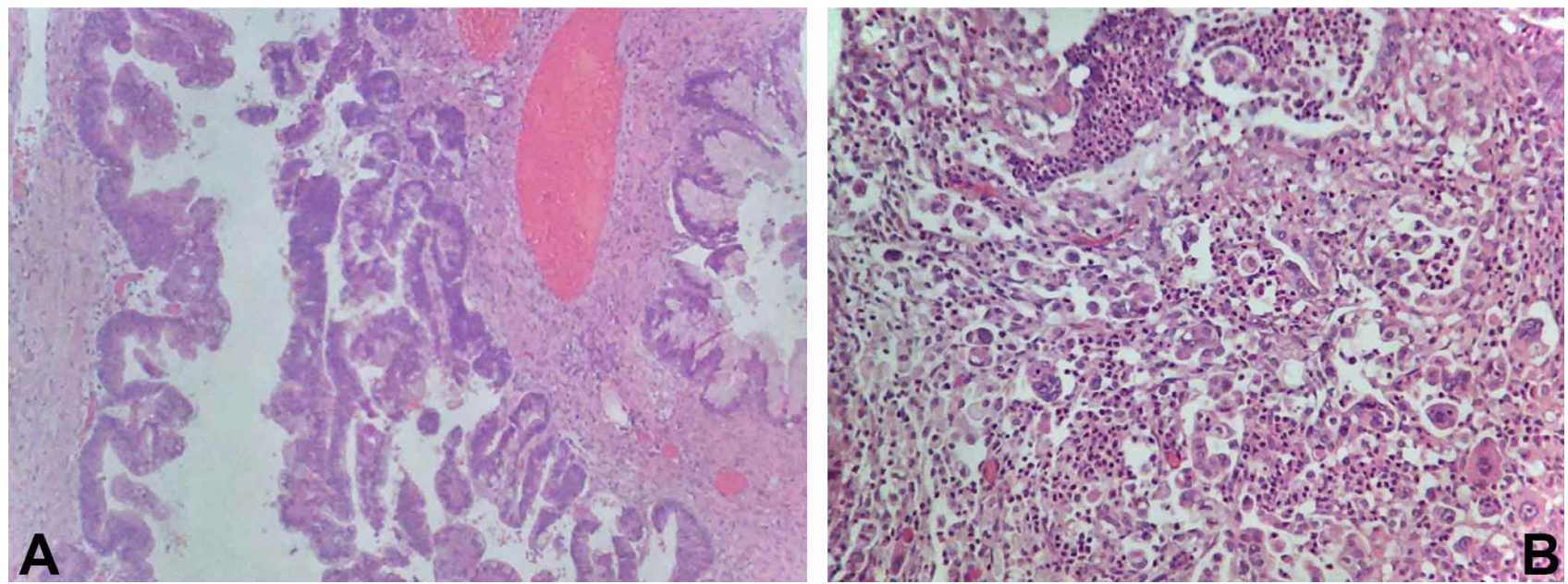

Fig. 5. Microscopía. Con tinción de HE, se puede verificar el componente arquitectural in situ (A: 4x); y microinvasor (B: 20x). 


\section{DISCUSIÓN}

El páncreas puede ser asiento de una variedad de lesiones quísticas, neoplásicas y no neoplásicas, que pueden ser asintomáticas o multisintomáticas. Con el desarrollo de las técnicas de imagen (TAC y RNM), se ha producido un incremento progresivo de hallazgos incidentales de este tipo de lesiones (prevalencia de $2,4 \%$ a $20 \%$ de las imágenes [Kucera et al., 2012; Sahani et al., 2013; Troncoso et al.]), algunas de las cuales pueden alcanzar grandes tamaños como ocurrió en este caso.

En relación al diagnóstico diferencial de los quistes pancreáticos, no hay biomarcadores de ADN, ARN o proteínas disponibles en sangre para uso clínico, que permitan diferenciar el tipo de quistes pancreáticos, o identificar displasia de alto grado de cáncer. Del mismo modo, no existe evidencia suficiente para apoyar el uso de marcadores tumorales como el CEA para diferenciar entre quistes pancreáticos (European Study Group on Cystic Tumours of the Pancreas).

Los factores de riesgo de progresión a cáncer que se han identificado son ictericia, presencia de un nódulo mural o de componente sólido $\geq 5 \mathrm{~mm}$; citología positiva, ó dilatación del conducto pancreático principal sobre 10 mm (European Study Group on Cystic Tumours of the Pancreas).

Desde la perspectiva anátomo patológica, se caracterizan macroscópicamente por masas quísticas grandes y redondas; de diámetros promedios entre 6 y $10 \mathrm{~cm}$; de superficie de corte uni o multilocular; con proyecciones papilares y nódulos murales, los que pueden corresponder aáreas de malignidad. Suelen estar llenos con abundante líquido mucoide claro, rodeado de una pared densa y fibrosa, con focos de calcificación (Zamboni et al., 1999). En cortes microscópicos se pueden verificar dos componentes histológicos diferentes: uno de ellos, consiste en una capa epitelial interna compuesta de células secretoras de mucina; y el otro, en un estroma celular denso, de tipo ovárico, que forma una banda de células en forma de huso densamente empaquetadas, bajo el epitelio neoplásico. La existencia de este estroma, puede ser útil para el diagnóstico, particularmente cuando el revestimiento epitelial está ampliamente desnudo (Kodiatte et al., 2016). Por otra parte, el componente epitelial consiste en células columnares productoras de mucina con diversos grados de atipia arquitectónica y nuclear, pudiendo ser plano o papilar; y el epitelio con displasia significativa a menudo está inmediatamente adyacente a epitelio benigno. Se ha visto que la posibilidad de encontrar focos displásicos o invasivos es mayor en las paredes y septos fibróticos engrosados, así como en los nódulos intramurales que se proyectan hacia la luz del quiste (pudiendo existir displasias de bajo grado, grado intermedio y de alto grado). Las displasias de alto grado (como el caso de la paciente en cuestión), se caracterizan por papilas ramificadas irregulares o estructuras cribiformes con importante atipias. De este modo, pueden aparecer carcinomas invasivos en alrededor de un $30 \%$ de los casos; los que pueden ser focales (confinado a la pared quística), o extenderse al tejido pancreático circundante u órganos adyacentes; no obstante lo cual, las metástasis linfonodales son raras (Terris; Kodiatte et al.).

Se ha propuesto una una clasificación en 3 subgrupos, que se ha utilizado para la tokma de decisiones terapéuticas y el pronóstico de estas lesiones. El cistadenoma mucinoso (representa aproximadamente el 65 $\%$ de estas lesiones), se caracteriza por una sola capa de células columnares mucinosas sin atipias; por ende es una lesión benigna para la que sólo basta su exéresis. La NQM proliferativa no invasiva (que representa el $30 \%$ ), compuesta por distintos grados de atipia, displasia, incluso carcinoma in situ, pero sin componente invasivo; por lo que también se considera curada con la exéresis total. Y, el cistadenocarcinoma mucinoso (aproximadamente el $5 \%$ restante), similar a los anteriores, pero con invasión del estroma subyacente, lo que lo transforma en un adenocarcinoma invasivo de comportamiento agresivo similar al del carcinoma ductal de páncreas, por lo que debe ser tratada con resección radical incluida linfadenectomía (Sarr et al., 2000; Jorba et al.; Tanaka et al.). Sin embargo, esta clasificación no considera aún las NQM con focos de micro invasión, que es el tipo de lesión que afectá a la paciente en cuestión.

La NQMP tiene indicación quirúrgica fundamentalmente por el riesgo de malignización (hasta $40 \%$ ). Factores a considerar para intervenir son: diámetro sobre 4 $\mathrm{cm}$ de, existencia de sintomatología; o cuando tienen factores de riesgo (nódulo mural), independientemente de su tamaño. Se han de considerer además, la localización de la lesión y las condiciones basales del paciente (Berland et al., 2010; Tanaka et al.; Farrell \& Fernández-del Casti1lo, 2013; European Study Group on Cystic Tumours of the Pancreas).

Los procedimientos quirúrgicos sugeridos para pacientes con NQMP son pancreatectomía distal en el 90-95 $\%$ (esto evita tratamiento incompleto del carcinoma invasivo), asociado a disección linfonodal y esplenectomía 
cuando existe evidencias de displasia de alto grado o cáncer, compromiso linfonodal en la TAC, compromiso tumoral de los vasos esplénicos o peripancreáticos; y citología o histología de adenocarcinoma o carcinoma endocrino (Lillemoe et al., 1999; Shoup et al., 2002; Carrère et al., 2007). En casos sin características sospechosas o bajo riesgo de malignidad, puede indicarse pancreatectomía distal con preservación esplénica con o sin preservación de vasos esplénicos. El acceso laparoscópico es factible pero su beneficio sobre un acceso abierto es comparable (European Study Group on Cystic Tumours of the Pancreas). En un estudio comparativo de reciente publicación, se verificó que la pancreatectomía con y sin preservación esplénica son similares; con la diferencia de mayor pérdida hemática y tiempo quirúrgico cuando se recurre a la esplenectomía. Por otra parte, conservar el bazo se asoció con evitar inmunizaciones periódicas en pacientes con preservación de bazo y de vasos esplénicos (Pais-Costa et al., 2019). Por otra parte, las enucleaciones y las pancreatectomías centrales son constituyen estrategias indicadas sólo para lesiones benignas o limítrofes, siempre que una biopsia contemporánea no muestre características de malignidad (Hackert et al., 2017).

Respecto de la adyuvancia indicada (FOLFIRINOX), cabe señalar que el rol de cada uno de sus componentes, permite incrementar los beneficios de cada uno de ellos por separado (el ácido folínico potencia la eficacia terapéutica del fluorouracilo; el 5-FU, es un antimetabolito que impide la duplicación del ADN; el Irinotecán, es un inhibidor de la topoisomerasa, por lo que evita la replicación del ADN; y el Oxaliplatino, derivado del platino, impide la reparación del ADN). Por otra parte, existe evidencia que demuestra una mejoría en la supervivencia al comparar este esquema con quimioterapia monodroga con gemcitabina (estándar de referencia en cáncer de páncreas), de 11,1 meses vs. 6,8 meses (Conroy et al., 2011).

Como limitaciones, se ha de considerar que se trata de la aportación de un caso único, y que la evidencia existente respecto de este tipo de casos es escasa, como para poder comparar resultados.

Entre las lecciones aprendidas de este caso clínico, cabe destacar el rol del seguimiento riguroso y periódico de las imágenes, para evitar el crecimiento exagerado de estas lesiones, que dificulta la exéresis quirúrgica completa, incrementa el riesgo de morbilidad y mortalidad postoperatoria; y reduce las expectativas de supervivencia.

Es complejo plantear una conclusión, sobretodo cuando se trata del reporte de un caso aislado de una pato- logía poco frecuente (al menos en esta forma de manifestación). Quizás, más que plantear una conclusión, comentar la lección aprendida respecto de la relevancia de la evolutividad imagenológica de una lesión tumoral; y la toma de decisiones oportunas que al respecto se han de considerar.

MANTEROLA, C.; NAVARRETE, A. \& BECKER, R. Giant mucinous cystic neoplasm of the pancreas undergoing pancreatectomy with splenic preservation. Int. J. Morphol., 38(6):1722-1728, 2020.

SUMMARY: Mucinous cystic neoplasm of the pancreas (MCNP) are variable types of tumors, which predominantly affect women (90-95\%), and usually appear incidentally in the 5th to 7 th decade of life. They are generally solitary lesions, with no involvement of the main pancreatic duct, rarely symptomatic and are associated with malignancy (10\% to $40 \%)$. The aim of this manuscript was to report a case of giant mucinous cystic neoplasm of the pancreas surgically treated and review the existing evidence regarding its morphological, therapeutic and prognosis characteristics. Patient: A 29 -year-old woman with a slightly symptomatic abdominal mass. The diagnosis was verified with ultrasound, computed axial tomography and magnetic nuclear resonance. The patient underwent surgery; an uneventful corporocaudal pancreatectomy with splenic preservation was performed. She was discharged on the fifth day, and has evolved adequately, without postoperative complications. MCNP is a complex lesion, which can be associated with malignancy, but the preoperative diagnosis of malignancy cannot be established with certainty. Its prognosis depends on early diagnosis and timely treatment.

KEY WORDS: Pancreatic Cyst / therapy; Pancreatic Neoplasms / diagnosis; Pancreatic Neoplasms / therapy; Pancreatectomy.

\section{REFERENCIAS BIBLIOGRÁFICAS}

Basturk, O.; Hong, S. M.; Wood, L. D.; Adsay, N. V.; Albores-Saavedra, J.; Biankin, A. V.; Brosens, L. A. A.; Fukushima, N.; Goggins, M.; Hruban, R. H.; et al. A revised classification system and recommendations from the Baltimore Consensus Meeting for Neoplastic Precursor Lesions in the Pancreas. Am. J. Surg. Pathol., 39(12):1730-41, 2015.

Berland, L. L.; Silverman, S. G.; Gore, R. M.; Mayo-Smith, W. W.; Megibow, A. J.; Yee, J.; Brink, J. A.; Baker, M. E.; Federle, M. P.; Foley, W. D.; et al. Managing incidental findings on abdominal CT: white paper of the ACR incidental findings committee. J. Am. Coll. Radiol., 7(10):754-73, 2010.

Burk, K. S.; Knipp, D. \& Sahani, D. V. Cystic pancreatic tumors. Magn. Reson. Imaging Clin. N. Am., 26(3):405-20, 2018.

Carrère, N.; Abid, S.; Julio, C. H.; Bloom, E. \& Pradère, B. Spleenpreserving distal pancreatectomy with excision of splenic artery and vein: a case-matched comparison with conventional distal pancreatectomy with splenectomy. World J. Surg., 31(2):375-82, 2007.

Conroy, T.; Desseigne, F.; Ychou, M.; Bouché, O.; Guimbaud, R.; Bécouarn, Y.; Adenis, A.; Raoul, J. L.; Gourgou-Bourgade, S.; de la Fouchardière, C.; et al. FOLFIRINOX versus gemcitabine for metastatic pancreatic cancer. N. Engl. J. Med., 364(19):1817-25, 2011. 
European Study Group on Cystic Tumours of the Pancreas. European evidence-based guidelines on pancreatic cystic neoplasms. Gut, 67(5):789-804, 2018.

Farrell, J. J. \& Fernández-del Castillo, C. Pancreatic cystic neoplasms: management and unanswered questions. Gastroenterology, 144(6):1303-15, 2013.

Gagnier, J. J.; Kienle, G.; Altman, D. G.; Moher, D.; Sox, H.; Riley, D. \& CARE Group. The CARE guidelines: consensus-based clinical case report guideline development. J. Clin. Epidemiol., 67(1):46-51, 2014.

Hackert, T.; Michalski, C. W. \& Büchler, M. W. Mucinous cystic neoplasms of the pancreas: a surgical disease. JAMA Surg., 152(1):26, 2017.

Hasan, A.; Visrodia, K.; Farrell, J. J. \& Gonda, T. A. Overview and comparison of guidelines for management of pancreatic cystic neoplasms. World J. Gastroenterol., 25(31):4405-13, 2019.

Hurtado-Pardo, L.; Cienfuegos, J. A.; Antoñanzas, J.; Benito, A.; Panadero, P.; Salguero, J.; Martí-Cruchaga, P.; Zozaya, G.; Valentí, V.; Pardo, F.; et al. Cystic tumors of the pancreas. An update of the surgical experience in a single institution. Rev. Esp. Enferm. Dig., 111(2):8793, 2019.

Jorba, R.; Fabregat, J.; Borobia, F. G.; Busquets, J.; Ramos, E.; Torras, J.; Lladó, L.; Valls, C.; Serrano, T. \& Rafecas, A. Neoplasias quísticas del páncreas. Manejo diagnóstico y terapéutico. Cir. Esp., 84(6):296306, 2008.

Kodiatte, T. A.; Burad, D. \& Rymbai, M. L. Clinicopathological features of intraductal papillary mucinous neoplasms of pancreas in a tertiary care center: a 14 year retrospective study. J. Clin. Diagn. Res., 10(8):EC10-3, 2016.

Kucera, J. N.; Kucera, S.; Perrin, S. D.; Caracciolo, J. T.; Schmulewitz, N. \& Kedar, R. P. Cystic lesions of the pancreas: radiologicendosonographic correlation. Radiographics, 32(7):E283-301, 2012.

Lillemoe, K. D.; Kaushal, S.; Cameron, J. L.; Sohn, T. A.; Pitt, H. A. \& Yeo, C. J. Distal pancreatectomy: indications and outcomes in 235 patients. Ann. Surg., 229(5):693, 1999.

Nimptsch, U.; Krautz, C.; Weber, G. F.; Mansky, T. \& Grützmann, R. Nationwide in-hospital mortality following pancreatic surgery in Germany is higher than anticipated. Ann. Surg., 264(6):1082-90, 2016.

Pais-Costa, S. R.; de Sousa, G. C. C.; Araujo, S. L. M.; Lima, O. A. T.; Martins, S. J. \& Torres, O. J. laparoscopic distal pancreatectomy with or without spleen preservation: comparative analysis of short and longterm outcomes. ABCD Arq. Bras. Cir. Dig., 32(3):e1461, 2019.

Sahani, D. V.; Kambadakone, A.; Macari, M.; Takahashi, N.; Chari, S. \& Fernandez-del Castillo, C. Diagnosis and management of cystic pancreatic lesions. AJR Am. J. Roentgenol., 200(2):343-54, 2013.

Sarr, M. G.; Carpenter, H. A.; Prabhakar, L. P.; Orchard, T. F.; Hughes, S.; van Heerden, J. A. \& DiMagno, E. P. Clinical and pathologic correlation of 84 mucinous cystic neoplasms of the pancreas: can one reliably differentiate benign from malignant (or premalignant) neoplasms? Ann. Surg., 231(2):205-12, 2000.

Shoup, M.; Brennan, M. F.; McWhite, K.; Leung, D. H. Y.; Klimstra, D. \& Conlon, K. C. The value of splenic preservation with distal pancreatectomy. Arch. Surg., 137(2):164-8, 2002.

Tanaka, M.; Fernández-del Castillo, C.; Adsay, V.; Chari, S.; Falconi, M.; Jang, J. Y.; Kimura, W.; Levy, P.; Pitman, M. B.; Schmidt, C. M.; et al. International consensus guidelines 2012 for the management of IPMN and MCN of the pancreas. Pancreatology, 12(3):183-97, 2012.

Terris, B. Pancreatic cystic tumors of mucinous type. Surg. Pathol. Clin., 4(2):537-52, 2011.

Troncoso, C. F.; Schiappacasse, F. G.; Villegas, A. P. \& Fernández, A. M. Neoplasia mucinosa gigante de páncreas: presentación de caso clínico y revisión de la literatura. Gastroenterol. Latinoam., 26(1):17-23, 2015 .

van Huijgevoort, N. C. M.; Del Chiaro, M.; Wolfgang, C. L.; van Hooft, J. E. \& Besselink, M. G. Diagnosis and management of pancreatic cystic neoplasms: current evidence and guidelines. Nat. Rev. Gastroenterol. Hepatol., 16(11):676-89, 2019.
Vege, S. S.; Ziring, B.; Jain, R.; Moayyedi, P. \& Clinical Guidelines Committee. American Gastroenterological Association Institute Guideline on the Diagnosis and Management of Asymptomatic Neoplastic Pancreatic Cysts. Gastroenterology, 148(4):819-22, 2015.

Warshaw, A. L. Conservation of the spleen with distal pancreatectomy. Arch. Surg., 123(5):550-3, 1988.

Zamboni, G.; Scarpa, A.; Bogina, G.; Iacono, C.; Bassi, C.; Talamini, G.; Sessa, F.; Capella, C.; Solcia, E.; Rickaert, F.; et al. Mucinous cystic tumors of the pancreas: clinicopathological features, prognosis, and relationship to other mucinous cystic tumors. Am. J. Surg. Pathol., 23(4):410-22, 1999.

Dirección para correspondencia:

Dr. Carlos Manterola Delgado

Departamento de Cirugía y CEMyQ

Universidad de La Frontera

Manuel Montt 112, oficina 408

Temuco

CHILE

Email: carlos.manterola@ufrontera.cl

Recibido : 18-05-2020

Aceptado: 19-07-2020 